\title{
Dynamics of crystal structure formation in spincoated colloidal films
}

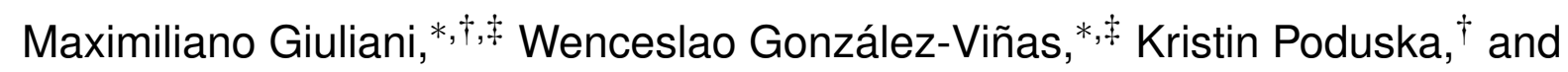
Anand Yethiraj ${ }^{*, \dagger}$
Department of Physics and Physical Oceanography, Memorial University, St. John's, NL,
Canada AlB 3X7, and Department of Physics and Applied Mathematics, University of Navarra,
Pamplona, Spain.

E-mail: mgiuliani@alumni.unav.es; wens@fisica.unav.es; ayethiraj@mun.ca

*To whom correspondence should be addressed

'Memorial University

+ University of Navarra 


\begin{abstract}
The spincoating of colloidal suspensions is an inherently non-equilibrium process that gives rise to highly reproducible, but polycrystalline, films with different symmetries depending on experimental parameters. In this study, we explore the transient dynamics of evaporative colloid spincoating for the first time, via a combination of high-speed imaging, atomic force microscopy, static photography and scanning electron microscopy. As the wet colloidal film thins and dries, we observe several symmetry transitions, while at the same time remarkably, the thinning rate (in non-dimensional time units) collapses to one universal curve for all rotation rates. We correlate static and dynamic measures of crossovers in ordering regimes, and obtain an estimate of the evaporation rate in the late stage of drying. We conclude that the thinning dynamics controls the local volume fraction and stress profiles, which in turn drives the structural transitions.
\end{abstract}

\title{
TOC Graphic
}

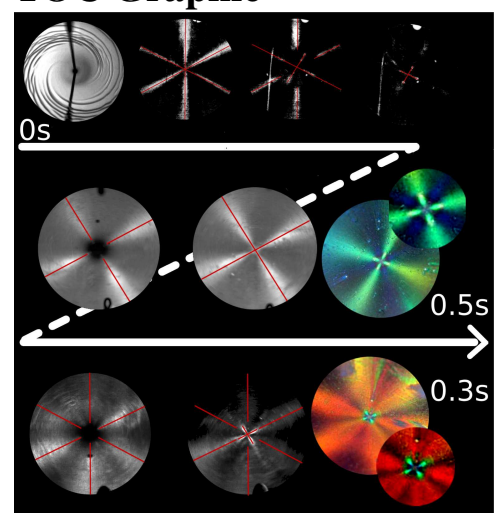

Keywords: Colloids, spin-coating, evaporation, phase transition, colloidal crystals. 
Colloidal self-assembly is a facile and promising method to making photonic crystals, ${ }^{1}$ but the control of defects is a challenge. Non-equilibrium approaches to colloidal self-assembly are likely crucial to the making of large-area colloidal crystals. ${ }^{2,3}$ Colloid spincoating has recently emerged as a highly robust and reproducible non-equilibrium method to make multilayer colloidal crystalline films. ${ }^{4-6}$ However, in spite of its robustness, the spincoating route to colloidal crystals is fraught with challenges. The highly uniform structural colors exhibited by these films have been shown to arise from a polycrystal where different crystallites exhibit long-range orientational order. ${ }^{7}$ In order to develop strategies to produce crystals with a greater degree of translational order, a deeper understanding of the dynamical mechanisms of structure formation is required.

While the dynamics of spincoating has been studied extensively and quantitatively in simple one-component fluids ${ }^{8,9}$ as well as in polymer solutions, ${ }^{10,11}$ the study of the dynamics of colloid spincoating has been limited to numerical studies of thinning rates. ${ }^{12}$ A recent work demonstrates that spincoating flows (in the absence of evaporation) control the local stress profiles, and drive crystallization when the Peclet number exceeds a critical value. ${ }^{6}$ Two questions that have remained unaddressed - how the dynamics interlinks with the emergence of different symmetries, and the role played by evaporation - are the focus of the experiments reported here.

We suspended silica particles ( $458 \pm 2 \mathrm{~nm}$ diameter) in methyl ethyl ketone (MEK) or acetone at different initial volume fractions $\left(\phi_{0}=5,10,15\right.$ and $\left.20 \% \mathrm{w} / \mathrm{w}\right)$ by procedures described elsewhere. ${ }^{7}$ Substrates were made from silicon wafers cut approximately to $25 \mathrm{~mm}$ x $25 \mathrm{~mm}$, rinsed with ethanol (96\%) and distilled water, dried by blowing of $\mathrm{N}_{2}$ gas, and stored in a clean box to avoid contamination until the moment of performing the experiment. Each experiment consisted of pipetting in continuous flow ("pouring") $40 \mu \mathrm{L}$ of suspension on the substrate spinning at a constant angular speed. The spinning was ceased in 10 to 15 seconds when the substrate was completely dry.

Dynamics of the spincoating process was obtained with a high speed camera at a rate of 1000 frames per second. A 300W tungsten bulb was used as light source in two different lighting setups (Figure 1). To visualize long-range order in both the fluid (thinning) and dried states, the light 


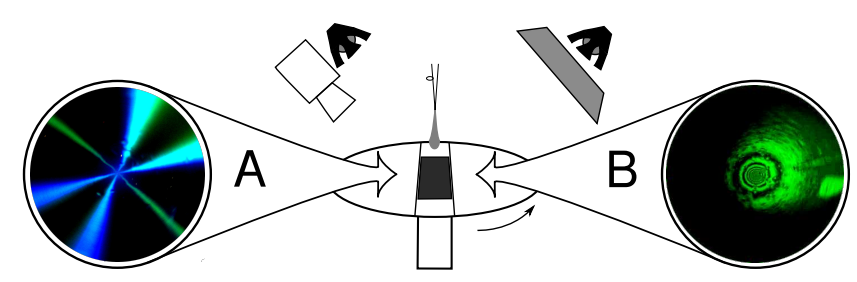

Figure 1: Experimental setups showing different lighting configuration that, A: magnifies contrast of the long range orientational order, B: enables the visualization of thickness in the fluid (band pass filter in the lens setup not shown).

source was located next to the camera (configuration A in Figure 1). Spatial and temporal thickness variations during the fluid stage were visualized (configuration B in Figure 1) via interference rings. In this case the light source was placed such that the specular reflection of the bulb was observed by the camera; a band pass filter set the imaging wavelength to $\lambda=540 \mathrm{~nm} \pm 40 \mathrm{~nm}$ and a diffuser placed in front of the light source homogenized the illumination.

Radial thickness profiles of the colloidal films were determined by AFM scanning using a method similar to that reported by Arcos et al. ${ }^{7}$ Static optical images of the dried samples (taken with a digital camera and the built-in flash as lighting) were used to evaluate the macroscopic long range order present in the colloidal crystal. Colloidal structure on a single-particle scale was obtained by SEM imaging of the top layer.

We begin with our observations of the transient dynamics. Using high-speed imaging and the lighting configuration A (Figure 1), we are sensitive to changes in long range orientational order via the appearance of symmetries in the optical reflections. In all experiments (at varying rotation speeds) a common dynamics is observed in the fluid phase. While the results presented here pertain to experiments using MEK as solvent, similar results were found with acetone as solvent. This phase is characterized (Figure 2(a)) by a set of von Kármán spirals ${ }^{13,14}$ that disappear when no more suspension is pipetted into the rotating substrate. Long range orientational order with 6-fold symmetry extending over the whole surface is observed immediately after pouring of the suspension ceased. This symmetry (Figure 2(b)) prevails for at least half of the duration between pipetting and drying. Next, there is a transition to 4-fold symmetry (Figure 2(c)). This has been observed in dried structures $5,7,15$ but it has never before been observed in the wet phase. The 4- 


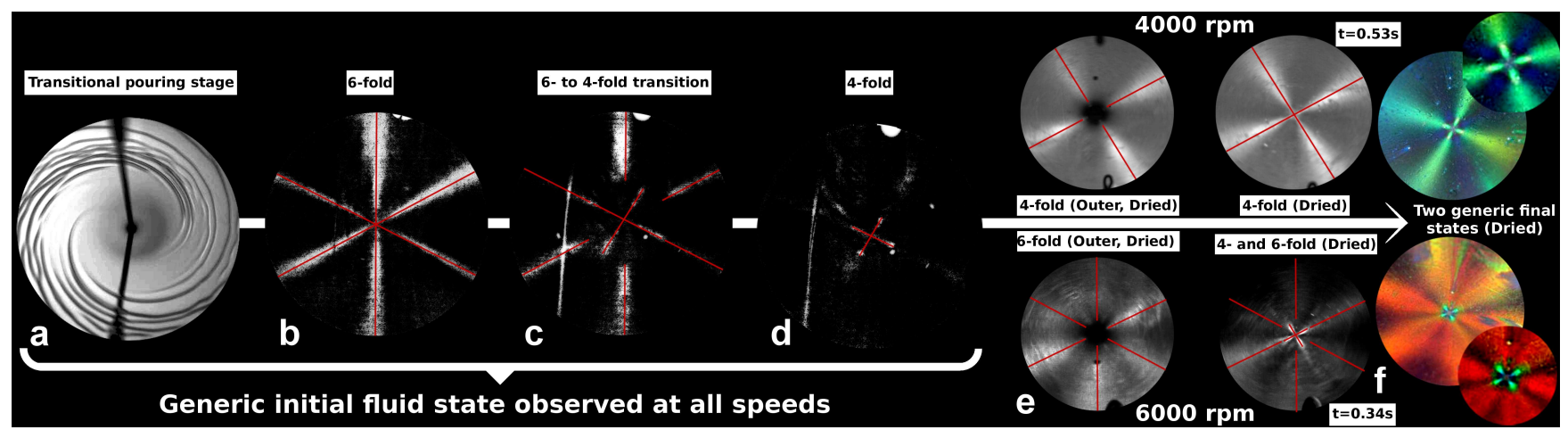

Figure 2: A typical sequence illustrates the transient dynamics of long range orientational order. (a) When the suspension is pipetted onto the rotating substrate, a set of von Kármán spirals appear. These spirals dissipate soon after the pipetting ceases. (b) With the disappearance of the spirals, 6-arm symmetry is immediately observed to be extended along the whole surface of the substrate. (c) This order prevails for relatively long times but finally transitions to 4-fold symmetry. (d) The 4-arm pattern rapidly shrinks towards the center. (e) The drying begins on the periphery and the drying front (the interface between bright and dark regions) propagates radially towards the center, revealing the final dried symmetry. The wet region loses its 6- or 4-arm order in the milliseconds prior to the arrival of the drying front. (f) The dynamics in the fluid stage for varying experimental conditions (either decreasing concentration or increasing angular speed of the substrate) is qualitatively identical and quantitatively universal (see text). However, the dried structure presents two distinct symmetries depending on experimental conditions: pure 4-fold symmetry at low angular speeds or high concentration (top right, 20\%, $4000 \mathrm{rpm}$, MEK solvent) and mixed structures, e.g center 4-fold and the outer area 6-fold, at high angular speeds or low concentration (bottom right, $20 \%, 6000 \mathrm{rpm}$, MEK solvent). Real color version of generic final state are shown at the rightmost of the figure. Red lines are drawn on top of arms as a guide to the eye. The commonality in fluid structures, but the two kinds of dried structures indicates that the drying front might erase all memory of the structure observed prior to drying. Snapshots were digitally enhanced to improve visualization. An entire movie of this transient dynamics can be found in the Supporting Information section.

fold order encompasses the whole surface of the substrate for a short time (e.g. 10 and 100ms at 6000 and $2000 \mathrm{rpm}$ respectively) before shrinking towards the center (Figure 2(d)). At this point complete drying of the structure is observed. In all cases, this drying starts from the edges of the substrate and is directed inwards defining a circular drying front whose diameter decreases with time (Figure 2(e)). Studies of these transient dynamics also show for the first time that the drying front is preceded by disordering, i.e. the disappearance of the symmetric reflections milliseconds prior to drying. A movie of the experiment is available in the Supporting Information section.

The dried state is characterized by two cases that are distinguished through the symmetry ob- 
served in the outer region of the samples (Figure 2(f)). The center presents 4-fold symmetry in all cases, while the outer region shows either 4-arms (low rpm) or 6-arms (high rpm) which are broader than the central ones. The transition from 4- to 6-fold structures takes place at 6000 or $7000 \mathrm{rpm}$, depending on the ambient temperature. Upon lowering the initial particle concentration from $\phi_{0}=20 \%$ to $5 \%$, a similar 4 - to 6-fold symmetry transition was observed at fixed angular speed $(3000 \mathrm{rpm})$ at a concentration of $10 \%$.

As reported previously, ${ }^{7}$ the symmetries observed macroscopically by static photography corresponded to $3 \mathrm{D}$ close packing with either square or hexagonal arrangement in the substrate plane (seen via SEM images of the top surface). Near the center the SEM images reveal a well defined orientational correlated square structure for all experimental conditions, except $\phi_{0}=5 \%$ where sub-monolayer coverage of the substrate is encountered. The three distinct dependencies - radial dependence, rotation rate dependence and concentration dependence - of the symmetry transitions likely all result from a transition to substrate-induced ordering as the film thickness is decreased. We thus look at the dynamics and statics of film thickness next.

We begin with the study of transient film-thinning dynamics in a region comprising radii between $1 \mathrm{~mm}$ and $5 \mathrm{~mm}$ from the center of rotation. Using the lighting configuration B depicted in Figure 1(video 2 in Supplementary Information), interference fringes allows us to observe the thinning process in the fluid state using a method conceptually similar to that reported by Birnie $e t$ $a l .{ }^{8}$ and by Heriot et al. ${ }^{16}$ (Figure 3(a)). We measured the frequency with which Newton rings pass a fixed radial distance. It was observed that this frequency was independent of the radial position, but changed rapidly with time. A standard Newton fringes calculation $\left(\left\langle n_{\mathrm{eff}}\right\rangle=1.42, \lambda=540 \pm 40\right.$ $\mathrm{nm}$ ) indicates a thickness change of $190 \pm 14 \mathrm{~nm}$ between consecutive bright rings. From this frequency, the thinning rate was calculated (details in Supplementary Information). This is shown in Figure 3(b), where the abscissa represents a rescaled (non-dimensional) time $\left(\tau=\omega\left(t-t_{\mathrm{dry}}\right)\right)$ and the ordinate a thinning rate also in re-scaled time units ( $\mu m$ per rad). Remarkably, we find that this rescaling collapses all curves for all rotation rates.

Figure 3(a) (right) shows a sharp decrease in the reflected light in the sample periphery (equiv- 


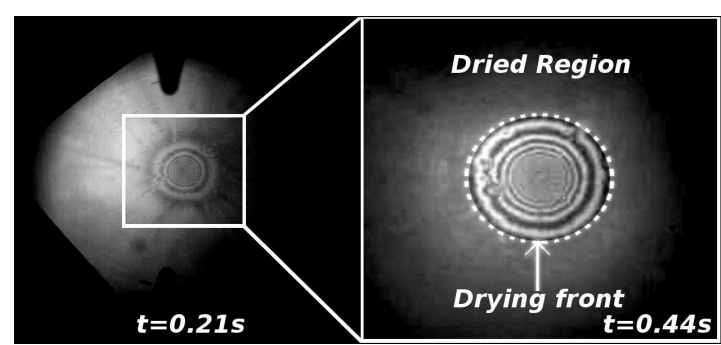

(a)

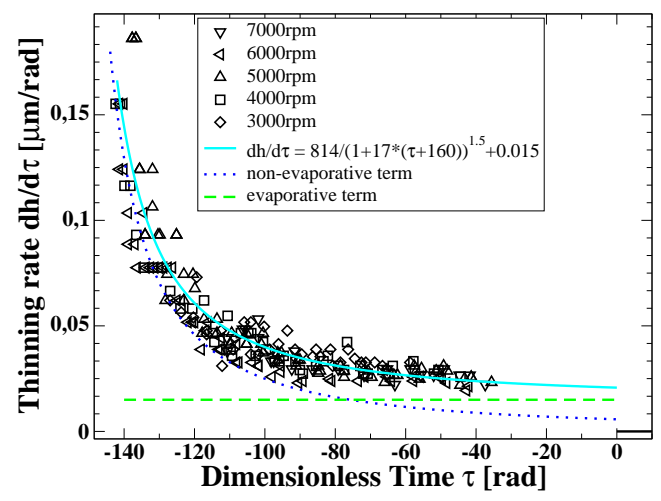

(b)

Figure 3: Universality in transient film-thinning dynamics, lighting configuration B. (a) Pattern of alternating rings observed during thinning $(\omega=5000 \mathrm{rpm}=523.6 \mathrm{rad} / \mathrm{s}$, MEK solvent $)$ at 0.21 and 0.44 seconds. The last image, $\mathrm{t}=0.438 \mathrm{~s}$, shows only the inner region for enhanced detail of the drying front transition. (b) The derivative of the thickness as a function of time measured from the alternating ring pattern (at radial distances from 1 to $5 \mathrm{~mm}$ ). A rescaling of time $\left(\tau=\omega\left(t-t_{\text {dry }}\right)\right.$ ) leads to a collapse of data at all rotation rates. $\tau=0$ is the moment when the sample completely dries. The results are well fit by the function in Eq. (1). Plotting the two terms of the fit (nonevaporative, dotted line and evaporative, dashed line) separately, we se that evaporation likely dominates for $\tau>80 \mathrm{rad}$.

alent to the sharp increase in the scattered light in Figure 2), indicating the drying of the structure. The radial position of the dry-wet interface (i.e. the distance of the drying front to the center of rotation) is plotted as a function of non-dimensional time $\tau$ in Figure 4(a) for different rotation rates. In all the experiments there are two well-defined regimes: an "early stage of drying" regime where typical curves at different $\omega$ do not collapse, and a "late stage of drying" regime, where they do collapse. Linear fits of both asymptotic regimes allow us to extract the corresponding characteristic speeds. Figure 4(b) shows these speeds (in rescaled time units) for each regime as a function of rotation rate. While in the outer region (corresponding to early stages of drying), the absolute value of the front speed increases with the rotation speed, in the central region (corresponding to 


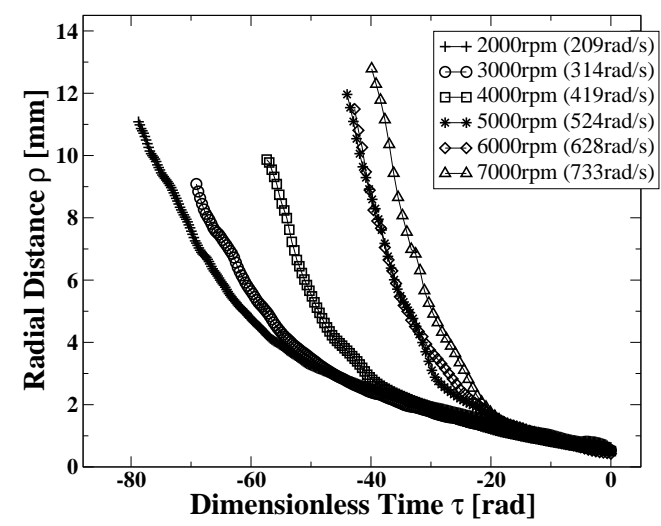

(a)

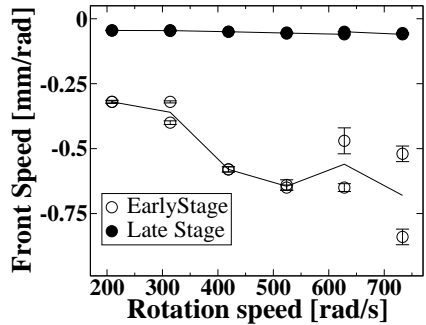

(b)

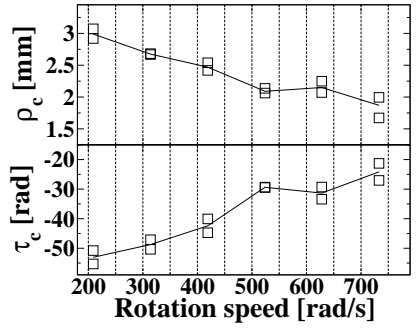

(c)

Figure 4: (a) Drying front radial position as a function of non-dimensional time (MEK solvent, $\phi_{0}=20 \%$ ). (b) Asymptotic slopes for early and late stages of the dynamic position of the drying front versus time. (c) Time and radial crossover of asymptotic slopes as a function of the rotation rates of the substrates. The lines joining the data points are visual guidelines.

late stages of drying) the drying front speed does not depend on the substrate speed. The transition point $\left(\rho_{c}, \tau_{c}\right)$ between dynamical regimes was defined via the crossover between the two asymptotic fits. The radial $\left(\rho_{c}\right)$ and temporal $\left(\tau_{c}\right)$ component of this crossover are shown in Figure 4(c) (top and bottom). A decrease in the radial component and an increase in the temporal component is observed as the rotation speed of the substrate is increased. Since $\tau=0$ corresponds to $t=t_{\mathrm{dry}}$, a less-negative $\tau_{c}$ implies that the crossover occur at later times. All the experiments described in this work were carried out at ambient temperatures $\left(20^{\circ} \mathrm{C}\right)$ for two solvents (MEK and acetone) which differ primarily in viscosity $(0.42 \mathrm{mPa} . \mathrm{s}$, and $0.31 \mathrm{mPa} . \mathrm{s}$ respectively) and in equilibrium vapor pressure (71 and $181 \mathrm{mmHg}$ respectively). The results from acetone have not been reported, for clarity. However, all the results presented, including the two-stage dynamics, are valid for both solvents.

Finally, the thickness of the colloidal crystal was measured using atomic force microscopy. Following techniques described elsewhere ${ }^{7}$ a scratch was made through the center of the structure and the height difference between the substrate and the top layer was determined as a function of radial position. The heights showed a bimodal distribution with one peak at the substrate location and the other on the top layer of the colloid sediment. They were fitted by gaussians and the 
difference between the mean of those fits was taken as the thickness of the structure.

In Figure 5(a) we present the thickness profiles of the crystals obtained (MEK suspensions). At the same speed, acetone profiles (not shown) are thicker. In all cases thickness profiles present a plateau in the outer region and an increase as we get closer to the center (radial distance $\rho=0$ ). All thickness profiles have an envelope similar to a bell function with a global maximum at the center of rotation.

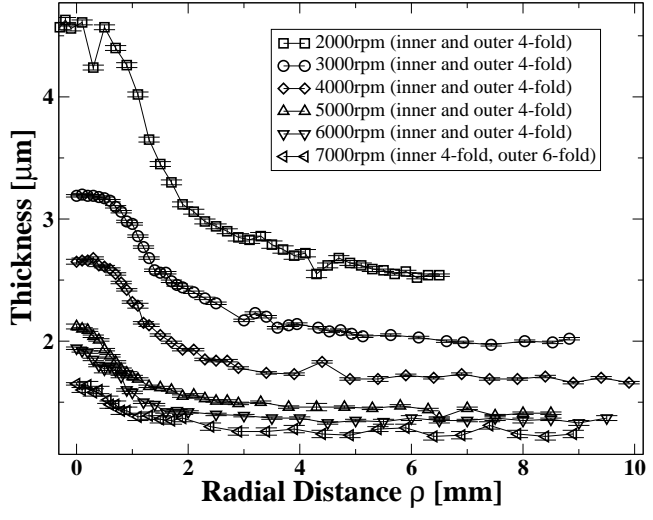

(a)

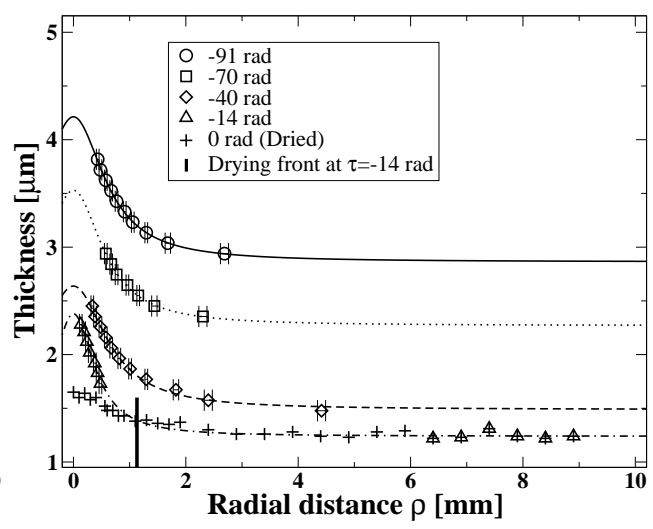

(b)

Figure 5: (a) AFM thickness profiles at different rotation rates(MEK solvent, $\phi_{0}=20 \%$ ). (b) Thickness profiles obtained at $7000 \mathrm{rpm}$ from the transient film-thinning dynamics at different nondimensional times. All thickness profiles show a similar trend. Lines are for visual guidance only. The vertical bar marks the position of the drying front at $\tau=-14$ rad extracted from Figure 4(a).

We can compare the static thickness profiles obtained by AFM with optical thickness profiles extracted from the ring-like interference patterns like the one shown in Figure 3(a) ${ }^{1}$. In Figure 5(b) we show thickness profiles obtained at different dimensionless times. It is clear that the shape of the fluid surface in the inner region, (i.e. $\rho<1 \mathrm{~mm}$ ) is very similar for $\tau=-91,-70$ and $-40 \mathrm{rad}$, but different for $\tau=-14$ rad (when the outer region is dry). This is consistent with observations (results of Figure 3(b)) that thinning rates (measured prior to complete drying, $\tau<-40$ rad) were independent of the radial distance.

Our study of transient dynamics (Figure 2) shows that both kinds of previously reported long

\footnotetext{
${ }^{1}$ Although the interference fringes do not allow determination of absolute thickness for the fluid phase, a reasonable (upper) limit is determined by utilizing the situation where the center presented rings while the outer region was already dried (see Supplementary Information for details). The other fluid thicknesses were determined using this situation as a reference.
} 
range orientationally ordered structures are found in a single system. On one hand, the fluid phase presents 6-fold symmetry. ${ }^{4}$ On the other hand, the final static structure after drying exhibits two different symmetries (4- and 6-fold) that were previously seen in evaporative spincoating. $5,7,15$ Remarkably, however, we find other symmetry transitions in the transient dynamics. Summarizing the results, we observe four different phases:

- Phase I: 6-fold symmetry (Figure 2 (b)). This is identical to the phenomenon reported by Jiang et al., ${ }^{4}$ where the inter-particle separation reported was $1.41 \sigma$ ( $\sigma$ is the particle diameter). Further assuming vertical compression Jiang et al. deduced a particle volume fraction of $\approx 52 \%$.

- Phase II: A transition to 4-fold symmetry (Figure 2 (c)). This has not been reported in the fluid phase before.

- Phase III: Loss of 4-arm symmetry in the outer region, milliseconds before drying (Figure 2 (d) and (e)). This again is new, and is specific to evaporative spincoating (and observable only because of the high-speed imaging). Shear-induced melting in a non-closed-packed crystal was first observed by Ackerson and Clark. ${ }^{17}$ What we observe is likely shear-induced melting driven by the disturbance preceding the drying front.

- Phase IV: dried close-packed structures with mixed outer/inner (4/4 or 6/4) symmetries.

In the fluid phase (phase I) the shear stress $\sigma \sim \rho_{\mathrm{MEK}} \omega^{2} \rho h$, where $\omega=500 \mathrm{rad} / \mathrm{s}, h \approx 1 \mu \mathrm{m}$ and density $\rho_{\text {MEK }} \approx 800 \mathrm{~kg} / \mathrm{m}^{3}$. The radial distance $\rho$ can be taken to be $1 \mathrm{~mm}$ in the inner region and 5-10 $\mathrm{mm}$ in the outer region. The Peclet number $P e \sim \frac{\sigma a^{3}}{k_{B} T}$ thus also increases with radial position from $0.8-8$. This corresponds well to the findings in Shereda et al that shear-induced crystallization occurs when the Peclet number is $\mathrm{O}(1)$.

The origin of phase II appears to coincide with times where the role of evaporation becomes more dominant in the thinning rate (see Figure 3(b)): i.e. both the radial variation in Peclet number and evaporation must be taken into account. 
The disordering in Phase III is a more challenging question. Here, the dry-wet interface provides a perturbation to the shear stress profile imposed on the spinning fluid which induces disordering of a shear-induced crystal. The most direct consistency check for this is dynamical. Natural colloidal re-organization timescales are $t_{\text {Brownian }} \approx a^{2} / D>15 \mathrm{~ms}\left(a=0.25 \mu \mathrm{m}\right.$ and $\left.D<\frac{k_{B} T}{6 \pi \eta a}\right)$. This timescale is a lower limit because $\eta_{\mathrm{eff}} \approx 10 \eta$ at $\phi=0.5$. The complete disappearance of the order occurs over $\approx 10 \mathrm{~ms}$. The characteristic length- and time-scales for front motion in the movie shown are approximately $1 \mathrm{~mm}$ and $10 \mathrm{~ms}$ respectively. For thin films in the outer region prior to drying, the volume fraction will increase rapidly: the Lindemann criterion ensures that for thin enough films, the resulting change in lattice spacing will be large enough so as to result in crystal melting. There is not enough time before the front arrives for re-crystallization at higher packing. Drying on the other hand forces local ordering at the drying front.

Another important aspect of the dynamics over times spanning Phases I to III is the existence of an universal behavior in the thinning rate as a function of dimensionless time (Figure 3(b)). We attempted to fit the experimental data to a phenomenological form inspired by the thickness vs. time equations for a simple fluid, ${ }^{18}$ but forcing the functional form to obey the experimentallyobserved time scaling: $h=h_{0} /\left(1+\alpha\left(\tau-\tau_{0}\right)\right)^{0.5}$. This functional form was not a good fit to the data near $\tau=0$ (not shown). Nevertheless, following Birnie et al. ${ }^{8}$ and Meyerhofer ${ }^{9}$ a constant can be added to the thinning rate to properly take into account the evaporative phase:

$$
-d h / d \tau=\left\{\begin{aligned}
\left(0.5 \alpha h_{0}\right) /\left(1+\alpha\left(\tau-\tau_{0}\right)\right)^{1.5}+\varepsilon & \text { if } \tau<0 \\
0 & \text { if } \tau \geq 0
\end{aligned}\right.
$$

This modified form is in excellent agreement with our measurements, with fitting parameters: $\alpha=17 \mathrm{rad}^{-1}, h_{0} \approx 96 \mu \mathrm{m}, \tau_{0}=-160 \mathrm{rad}$ and $\varepsilon=0.015 \mu \mathrm{m} / \mathrm{rad} . h_{0}$ represents the starting height of the film at the end of the transient von Kármán spirals and $\tau_{0}$ the corresponding time. We find therefore that the dimensional evaporation rate $e=\varepsilon \omega$ has a linear dependence on $\omega$ : for $\omega=500 \mathrm{rad} / \mathrm{s}, e=7.5 \mu \mathrm{m} / \mathrm{s}=0.75 \mu \mathrm{L} / \mathrm{s} / \mathrm{cm}^{2}$. In addition, this universal behavior looks similar to (and can in principle be compared with) the numerical thinning rates calculated by Rehg and 
Higgins. ${ }^{12}$

Having considered the dynamics in the fluid phase, we next consider the movement of the drying front (Figure 4(a)). In all cases studied, the speed at which the interface moves towards the center presents two distinct dynamical regimes (Figure 4(b)): an early stage where front speeds are large and increase in magnitude with increasing rotation speed, and a late stage where front speeds are small and invariant with rotation speed. The radial crossover from sharp arms near the center of rotation to the broader arms far from it correspond reasonably well with the dynamical crossover $\rho_{c}$ (Figure 4(c)) - the ratio of these two lengthscales is approximately 0.5 (with a standard error of \pm 0.25 ). Indeed, the much narrower arms in the central than in the outer region (Figure 2 ) is also consistent with the front speeds measured (Figure 4(b), slow/fast front speeds in the central/outer regions): the slower the front motion, the sharper the arms.

Finally, we compared static thickness profiles with the dynamic thickness profiles. We found that the thickness of the film is inhomogeneous even in the fluid state (Figure 5(b)), and there is always a maximum in thickness at the center of spinning. This inhomogeneity does not give rise to radial variations in the symmetry in, for example, phase I (see Figure 1 and Figure 2(b)), while in the dried state, the change in thickness towards the center corresponds to a change in morphology (Figure 2(f)).

That the fluid is indeed not planar is shown clearly in both the dynamical optical and the static atomic force microscopy measurements (Figure 5). It has been noted quite early by Acrivos et $a l .{ }^{19}$ that it is much harder to planarize a non-Newtonian fluid. As pointed out by Rehg and Higgins, ${ }^{12}$ the functional relationship between viscosity and concentration appears to play a much more significant role in spincoating of colloidal than in polymeric suspensions and the simple assumptions made for predicting coated film thickness in polymeric systems are inadequate for colloidal suspensions. The Eq. (1) is a phenomenological alteration of the Meyerhofer formula. ${ }^{9}$

At the late stage of drying $(\rho<1 \mathrm{~mm})$, we may assume a disk-like shape for the suspension, since the maximum value of $d h / d \rho \approx 10^{-3}$ (Figure 5). Then, the rate of change in the volume of wet suspension is $d V / d t=2 \pi \rho(d \rho / d t) h+\pi \rho^{2}(d h / d t)$. Note that all the quantities in the above 
equation have been obtained in this experiment. The term $d \rho / d \tau$ is obtained from the asymptotic fitting of the late stage (see Figure $4(\mathrm{~b})$ ). The thinning rate $d h / d t$ in the second term in this equation is obtained from the fit to the thickness profile for $\tau=-40 \mathrm{rad}$ (Figure 3(b)). However, the first term in this equation is the dominant one, i.e. the crystallization front is parallel to the outer meniscus of the wet film. In Figure 6 the rate of volume change is plotted as a function of the radial distance for the late stage of the drying front dynamics (Figure 4(a)). For this calculation the thickness profile $h(\rho)$ was obtained from the static thickness profile (Figure 5(a)).

This calculation show a linear decrease of the evaporation rate as we get closer to the center of rotation. Linear dependence of the $d V / d t$ suggests that the capillary flow of solvent through the interface (i.e. the $2 \pi \rho(d \rho / d t) h$ term in $d V / d t)$ is the primary contributor to the evaporation rate.

Why does the dried inner region always exhibit 4-arm order? When the film is thin enough the substrate will govern the ordering and 6-arm order is the result. For thicker films the radial drying front dominates, and other orientations are possible. The increasing Peclet number with increasing radius probably also plays a role in generating the mixed symmetries.

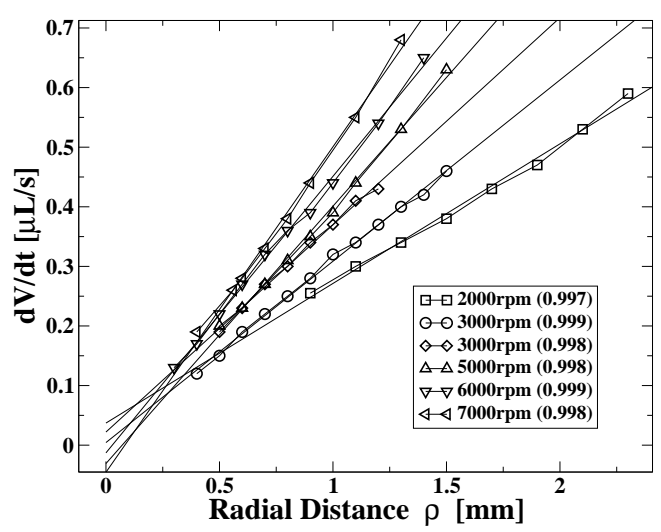

Figure 6: The rate of volume change $d V / d t$ (calculated from experimentally obtained parameters $\rho, d \rho / d t, d h / d t$ and $h)$ in the inner region as the drying front advance towards the center.

In summary, we have studied the high-speed dynamics of evaporative spincoating and found new structural transitions. In spite of the existence of these structural transitions, we find that the thinning dynamics exhibits no breaks at these transitions, and has a universal scaling form for all rotation speeds. Based on this, we suggest that the thinning dynamics controls the local 
volume fraction and stress profiles, which in turn drives the structural transitions. In addition, our quantitative measurements will enable detailed comparisons with numerical simulations of the spincoating of colloidal suspensions in the presence of evaporative flows.

\section{Acknowledgement}

The authors thank Professor Claude Daley (Department of Engineering, Memorial University) for a generous loan of his high-speed camera. This work was supported by NSERC, Spanish MEC (Grant No. FIS2008-01126), and Ministerio de Educación (Gobierno de Navarra). M.G. acknowledges support from the Asociación de Amigos de la Universidad de Navarra.

\section{Supporting Information Available}

Complete movies of the experiment on each configuration and detailed description of the measuring procedures for thickness related data (fluid).

This material is available free of charge via the Internet at http://pubs.acs.org/.

\section{References}

(1) Wijnhoven, J. E. G. J.; Vos, W. L. Preparation of Photonic Crystals Made of Air Spheres in Titania. Science 1998, 281, 802-804.

(2) Norris, D. J.; Arlinghaus, E. G.; Meng, L.; Heiny, R.; Scriven, L. Opaline Photonic Crystals: How Does Self-Assembly Work? Adv. Mater. 2004, 16, 1393-1399.

(3) Khanh, N. N.; Yoon, K. B. Facile Organization of Colloidal Particles into Large, Perfect One- and Two-Dimensional Arrays by Dry Manual Assembly on Patterned Substrates. J. Am. Chem. Soc. 2009, 131, 14228-14230.

(4) Jiang, P.; McFarland, M. Large-Scale Fabrication of Wafer-Size Colloidal Crystals, Macroporous Polymers and Nanocomposites by Spin-Coating. J. Am. Chem. Soc. 2004, 126, 13778. 
(5) Mihi, A.; Ocaña, M.; Míguez, H. Oriented Colloidal-Crystal Thin Films by Spin-Coating Microspheres Dispersed in Volatile Media. Adv. Mater. 2006, 18, 2244-2249.

(6) Shereda, L.; Larson, R.; Solomon, M. Local Stress Control of Spatiotemporal Ordering of Colloidal Crystals in Complex Flows. Phys. Rev. Lett. 2008, 101, 038301.

(7) Arcos, C.; Kumar, K.; González-Viñas, W.; Sirera, R.; Poduska, K. M.; Yethiraj, A. Orientationally Correlated Colloidal Polycrystals without Long-Range Positional Order. Phys. Rev. E 2008, 77, 050402R.

(8) Birnie III, D. P.; Manley, M. Combined Flow and Evaporation of Fluid on Spinning Disk. Phys. of Fluids 1997, 9, 870-875.

(9) Meyerhofer, D. Characteristics of Resist Films Produced by Spinning. J. Appl. Phys. 1978, 49, 1949-1959.

(10) Bornside, D. E.; Macosko, C. W.; Scriven, L. E. Spin Coating: One-Dimensional Model. J. Appl. Phys. 1989, 66, 5185-5193.

(11) Ohara, T.; Matsumoto, Y.; Ohashi, H. The Film Formation Dynamics in Spin Coating. Phys. of Fluids A 1989, 1, 3993-3997.

(12) Rehg, T.; Higgins, B. Spin Coating of Colloidal Suspensions. AIChe J. 1992, 38, 489.

(13) Wahal, S.; Oztekin, A.; Bornside, D. E.; Brown, R. A.; Seidel, P. K.; Ackmann, P. W.; Geyling, F. T. Visualization of a Gas Flow Instability in Spin Coating Systems. Appl. Phys. Lett. 1993, 62, 2584.

(14) Furukawa, H.; Tsutsui, H.; Aoi, K.; Watanabe, T.; Nakamura, I. Visual Study of Variations of Flow Patterns around a Disk in a Casing: Effects of Edge of the Disk. J. Phys.: Conference Series 2005, 220-227.

(15) Vermolen, E. Ph.D. thesis, Utrecht University, 2008. 
(16) Heriot, S. Y.; Jones, R. A. L. An Interfacial Instability in a Transient Wetting Layer leads to Lateral Phase Separation in Thin Spin-cast Polymer-blend Films. Nat. Mater. 2005, 4, 782786.

(17) Ackerson, B. J.; Clark, N. A. Shear-Induced Melting. Phys. Rev. Lett. 1981, 46, 123-126.

(18) Emslie, A. G.; Bonner, F. T.; Peck, L. G. Flow of a Viscous Fluid on a Rotating Disk. J. Appl. Phys. 1958, 29, 858.

(19) Acrivos, A.; Shah, M. J.; Petersen, E. E. On the Flow of a Non-Newtonian Liquid on a Rotating Disk. J. Appl. Phys. 1960, 31, 963. 